\title{
Intellectual Functioning in Schizophrenia, Depression and Normal Control
}

\author{
Dr. Anuradha Wats 1
}

\section{ABSTRACT:}

Background: Schizophrenia is one of the most severe and disabling of all mental illnesses. The diagnosis of schizophrenia invoices a constellation of signs and symptoms and impairment in occupational and social functioning. Mood disorders are also important \& common psychiatric illnesses that represent a major cause of disability and mortality worldwide.

Material and Methods: Sample of the study30 male and female Depression patients and a sample of 30 male and female schizophrenia patients was selected both from OPD. A control group of 30 persons from general population, matched according to age, sex, and education with schizophrenia patients, were taken as per Inclusion \& Exclusion criteria. The sample was selected using purposive sampling method. Samples were collected from P.G.I.B.M.S. Result: But post hoc analyses show that the schizophrenia and depressive groups differed significantly as the patients of depression had higher age mean than that of schizophrenia. Conclusion: Schizophrenia patients suffer from significant overall cognitive impairment; Have low concentration and inadequate visual organization. Depressive patients perform in a way similar to normal except in comprehension subtest

Keywords: Schizophrenia, Depression, WAPIS, VAIS, P.G.I

\section{INTRODUCTION:}

Cognition is what enables humans to function in everyday life personal, social and occupational. The ability to attend to things in a selective and focused way, to concentrate over a period of time, to learn new information and skills, to plan, to determine strategies for actions and to execute them, to comprehend language and to use verbal skills for communication and selfexpression and to retain information and manipulate it to solve complex problems are examples of mental processes that are referred to as cognitive function. All these abilities are impaired to some extent in individuals with schizophrenia and depression. This impairment inevitably has an impact on functional outcome of the illness [i.e. individual's ability to have social interactions, to hold jobs, and to care for themselves. Schizophrenia \& mood disorders are two important mental illnesses affecting psychosocial functioning and causing disability and cognitive deficits are prominent in both.

${ }^{1}$ Asst. Professor, P.G.I.B.M.S, Raipur

(C) 2015, A wats; licensee IJIP. This is an Open Access Research distributed under the terms of the Creative Commons Attribution License (http://creativecommons.org/licenses/by/2.0), which permits unrestricted use, distribution, and reproduction in any Medium, provided the original work is properly cited. 


\section{SCHIZOPHRENIA \& NEUROCOGNITIVE DEFICITS}

Schizophrenia is one of the most severe and disabling of all mental illnesses. The diagnosis of schizophrenia invoices a constellation of signs and symptoms and impairment in occupational and social functioning. Psychotic symptoms include delusions, prominent hallucinations, disorganized speech, or disorganized and catatonic behaviour. Negative symptoms include alogia, affective blunting, avolitionlasociality, and anhedonia. Finally, a separate domain of the illness that appears closely related to functional outcome involves the cognitive deficits of schizophrenia [Goldberg, Gold, et al., 1995; Green 1996; Green et al., 2000]. The range of cognitive deficits is broad and includes problems in perception, attention, memory, and problem solving [Green,. 2001].

Cognitive impairment is recognized as a core characteristic of schizophrenia (Heaton et. al., 1978; Seidman, 1983; Goldberg \& Seidman, 1991; Bilder et al., 1992). The study of cognitive processes has been an important avenue for linking the biological and social aspects of the illness (Hemsley, 1991). It is widely recognized that cognitive impairment, especially memory, is an integral part of the disease process (McKenna et al., 1991) and is associated with poor psychosocial functioning (Green, 1996). There has been considerable work to document the types of neurocognitive deficit in patients with schizophrenia. The presence of diffuse cognitive impairment has been documented, and patients with schizophrenia have been found to function one standard deviation or below the mean of healthy comparison subjects across a range of cognitive measures [Hoff, et al., 1992]. A Meta - analysis by Heinrichs and Zakaanis [Heinrichs et al., 1998] reviewed 204 studies and reported 22 mean effect sizes, looking at performance of patients with schizophrenia relative to healthy controls. Neurocognitive indices included general intellectual function, global and selective verbal memory, nonverbal memory, visual and auditory attention, executive function, language, spatial ability, motor performance, and inter hemispheric tactile transfer test performance. Moderate- to-large effect sizes (>0.60) were reported for all 22 indices, indicating that schizophrenia is characterized by broad - based cognitive impairment, with varying degrees of deficit in all ability domains measured by standard clinical tests. The largest effect sizes were for measures of standard clinical tests. The largest effect sizes were for measures of memory and attention. This finding is fairly consistent across studies. Torrey [Torrey et al., 2002] recently reviewed the literature examining first episode, never medicated patients with schizophrenia to determine whether deficits are present even early in the disease. Some of the same deficits including verbal memory and learning [Censits et al. 1997; Saykin et al., 1994], visual memory [Censits et al., 1997], abstraction [Censits et al., 1997], attention [Censits et al., 1997; Lussier et al., 2001; Schuepbach et al., 2002] and language abilities [Censits et al., 1997] have been found even in untreated, first episode patients.

It has been proposed that there are more specific deficits in schizophrenia that occur within the context of diffuse cognitive impairment [Saykin et al., 1991; Goldberg et al., 1987]. Several studies and meta - analysis have indicated that it is a disproportionate impairment in verbal declarative memory in schizophrenia over and above the global deficits [Saykin et al.,1991; 
Cirillo et al., 2003]. Saykin et al [Saykin et al., 1991] for example, described a specific impairment in memory and learning in unmediated patients with schizophrenia without a history of substance abuse

Thus, neurocognitive deficits generally are seen in patients with schizophrenia. Although there appears to be a group of patients who are impaired only minimally, most patients are characterized as having at least some impairment across a number of domains. Several studies identify selective impairments of a greater magnitude against a background of global impairment. Specifically, the cognitive deficits appear to be larger in areas of episodic memory and vigilance than in other cognitive areas e.g, immediate memory and vocabulary). The Neurocognitive deficits may be related especially to disorganized and negative symptoms and less with psychotic symptoms.

\section{MOOD DISORDERS AND COGNITIVE DEFICITS}

Mood disorders are also important \& common psychiatric illnesses that represent a major cause of disability and mortality worldwide. It is estimated that $8 \%$ to $20 \%$ of the population will experience a depressive episode at some point in their lives [Andreasen et al., 1995]. Of those individuals with symptoms so severe as to require hospitalization, $15 \%$ will go on to commit suicide. Mood disorders are characterized by disturbances in internal emotional state of an individual and are not limited to the external expression of emotional content. Two general conditions of mood disorder are clinical depression and bipolar affective disorder (BAD). BAD is marked by episodes of clinically significant impairment due to mania and depression. Although cognitive dysfunction in psychoses has classically been associated with schizophrenia, there is evidence that some bipolar patients show cognitive disturbances. Measurable decreases in attention, executive function, and recall memory have been observed in patients with mood disorders .In major depression, cognitive impairment can be severe and global, sometimes meeting criteria for dementia [Rabins et al., 1984].

Cognitive deficits within mood disorder have been studied extensively. Although results have not always been consistent, an overall pattern of specific impairments has become evident. In general, unipolar and bipolar patients have shown impaired performance in tests of attention, executive function, and memory.

Intelligence consists of various abilities that help us to adapt to life situations. It is an important aspect of cognitive impairment. People need preserved intelligence to cope with challenges of

life, to learn and relearn social and self-help skills. So, to see what aspects of intelligence are affected in patients of schizophrenia and depression this study was undertaken.

\section{NEED FOR THE STUDY :}

Studies reveal that cognitive deficits are not only the result of neuroleptic medications, they also are part of the psychopathology of schizophrenia. Patients of mood disorder are also found to be suffering from cognitive impairments. Attention and concentration, memory, executive functions 
etc. are found to be affected in both. Impairment of cognitive function is likely to have important functional consequences like interference with daily routine tasks and demands of meaningful occupational and social role functioning. Cognitive assessment also identifies individuals preserved abilities so that during rehabilitation remaining assets may be used to aid in compensating for those skills no longer available (Clipolotti \& Warrington, 1995; Green, 1998; Steffy, 1993). Since intelligence is at the base of these functions this study was undertaken to study the comparative profile of schizophrenia and depression patients, to help understand these conditions in a better way and thus in better management

\section{HYPOTHESIS}

1. There will be no difference in VAIS profile of depression, schizophrenia and normal groups.

2. There will be no difference in performance in WAPIS of depression, schizophrenia and normal groups.

\section{Alternative Hypotheses}

1. Patient groups will perform poorly on VAIS than normal groups.

2. Patient groups will perform poorly on WAPIS than normal groups.

The study was conducted at Post Graduate Institute of Behavioural and Medical Sciences, Raipur (C.G.). A sample of 30 male and female Depression patients and a sample of 30 male and female schizophrenia patients was selected both from OPD. A control group of 30 persons from general population, matched according to age, sex, and education with schizophrenia patients, were taken as per Inclusion \& Exclusion criteria. The sample was selected using purposive sampling method.

\section{Inclusion criteria (Depression patients): -}

- Patients in the age range of 18 yrs to $50 \mathrm{yrs}$.

- Patients diagnosed as having depression according to ICD-10 criteria.

- Right handed.

- Patient's cooperative for study.

- Exclusion criteria (Depression patients): -

- Major medical illness, organic mental disorders or substance related problems.

- Patients with other psychiatric illness.

- Patients with history of any neurological disorder or significant head injury.

- Inclusion Criteria:

- Patients diagnosed as having schizophrenia according to ICD-10 criteria.

- Right handed.

- Consenting and cooperating for study.

- Exclusion Criteria (Schizophrenia patients): -

- Persons with any other psychiatric disorder.

- Persons with history of any neurological disorder or significant head injury.

- Persons with any substance related problem, mental retardation or known organic brain disorder. 
Inclusion Criteria (Normal controls): -

- People from general population

- Those consenting for the study

- Exclusion Criteria (Normal controls): Persons scoring more than 12 on PGI Health Questionnaire

- Persons with any substance related problem, mental retardation or known organic brain disorder.

- Persons with history of any neurological disorder or significant head injury.

\section{TOOLS USED}

1. Socio-demographic and clinical data sheet.

2. Verbal Adult Intelligence Scale (Indian Adaptation )

3. WAPIS (Indian Adaptation, Prabha Ramalingaswamy, )

4. Handedness preference schedule (Mandal et al., 1992)

5. The Hamilton Rating Scale for depression (Hamilton, 1960).

6. Brief Psychiatric Rating Scale (Overall, G. E. \& Gorham, D. R., 1962)

7. PGI Health Questionnaire (S.K VERMA, N.N WIG \& D. PRASAD,1984)

\section{PROCEDURE}

Patients of Schizophrenia and Depression were selected from the OPD of PGIB\&MS. Normal group was selected from general population. Subjects meeting their respective inclusion and exclusion criteria were screened by using PGI health Questionnaire N-1 and those scoring more than 12 were excluded from the study. Information was gathered from all groups on semistructured socio-demographic data sheet. BPRS was used to assess the severity of the psychotic Symptoms in Schizophrenia Patients. HDRS was used to assess the severity of symptoms of Depression. To assess the intellectual functioning of the subjects Indian adaptation of Verbal intelligence test VAIS was for verbal intelligence, taken from PGI-BBD. For the assessment of performance intelligence, WAPIS (Indian adaptation) was used. Statistical analyses were done from the data thus obtained.

\section{STATISTICAL ANALYSIS:}

Data was statically analyzed using the descriptive analyses, Chi-square, ANOVA \& Post hoc analyses. Clinical demographic data was evaluated with descriptive statistical measures, which includes mean, standard deviation. Group differences were calculated by Chi Square.

- ANOVA was used for comparing the WAPIS and VAIS performances of depression and schizophrenic patients and normal group

- To see the differences among specific groups Post hoc analysis was done using LSD method. 


\section{RESULTS}

Table 1. Comparison of Age of Schizophrenia, Depression \& Normal groups

\begin{tabular}{|c|c|c|c|c|c|c|}
\hline \multirow[t]{2}{*}{ Variables } & \multicolumn{3}{|l|}{ Groups } & \multirow[t]{2}{*}{$\mathbf{F}$} & \multirow[t]{2}{*}{ Sig. } & \multirow{2}{*}{$\begin{array}{l}\text { Post } \\
\text { Hoc }\end{array}$} \\
\hline & $\begin{array}{l}\text { Depression } \\
\text { Mean } \pm \text { SD }\end{array}$ & $\begin{array}{l}\text { Schizophrenia } \\
\text { Mean } \pm \text { SD }\end{array}$ & $\begin{array}{l}\text { Normal } \\
\text { Mean } \pm \text { SD }\end{array}$ & & & \\
\hline Age & $29.60 \pm 10.60$ & $36.00 \pm 10.39$ & $33.77 \pm 12.51$ & 2.52 & .086 & $S<D$ \\
\hline
\end{tabular}

Table 1 shows the comparison of patients of Schizophrenia \& Depression with normal control group. The mean age of normal and schizophrenic \& depressive groups was 31.23(SD 9.62) years and 33.93(SD: 14.67) years respectively. In all the groups, subjects were within the age range 18 yrs to 55 yrs. Schizophrenia, Depression \& Normal groups didn't differ with respect to age as mean difference was statistically insignificant. But post hoc analyses show that the schizophrenia and depressive groups differed significantly as the patients of depression had higher age mean than that of schizophrenia.

Table 2. Shows the severity of depression in the depressed group

\begin{tabular}{|l|l|l|}
\hline H D R S & F & \% \\
\hline NONE & 0 & 0 \\
\hline MILD & 0 & 0 \\
\hline MODERATE & 5 & 16.7 \\
\hline SEVERE & 17 & 56.7 \\
\hline VERY SEVERE & 8 & 26.7 \\
\hline
\end{tabular}

Table 2 Shows the severity of depression in the depressed group. Majority (56.7\%) were severely depressed, $26.7 \%$ were very severely depressed and $16.7 \%$ were depressed upto moderate level. 
Table 3. Shows the comparison of WAPIS performance of Schizophrenia, Depression \& Normal groups

\begin{tabular}{|c|c|c|c|c|c|}
\hline \multirow[t]{2}{*}{ Variables } & \multicolumn{3}{|l|}{ Groups } & \multirow[t]{2}{*}{$\mathrm{F}$} & \multirow[t]{2}{*}{ Post Hoc } \\
\hline & $\begin{array}{l}\text { Depression } \\
\text { Mean } \pm S D\end{array}$ & $\begin{array}{l}\text { Schizophrenia } \\
\text { Mean } \pm \text { SD }\end{array}$ & $\begin{array}{l}\text { Normal } \\
\text { Mean } \pm \text { SD }\end{array}$ & & \\
\hline $\begin{array}{l}\text { PICTURE } \\
\text { COMPLETION }\end{array}$ & $8.57 \pm 9.23$ & $7.23 \pm 20.85$ & $9.23 \pm 7.26$ & $7.31 * *$ & $\mathrm{~S}<\mathrm{D}^{*}, \mathrm{~N}^{*}$ \\
\hline DIGIT SYMBOL & $5.53 \pm 8.03$ & $5.63 \pm 7.46$ & $9.27 \pm 4.02$ & $60.16 * *$ & $\mathrm{~S}<\mathrm{N}^{*}$ \\
\hline BLOCK DESIGN & $5.40 \pm 8.20$ & $5.83 \pm 14.31$ & $8.60 \pm 19.02$ & $48.39 * *$ & $\mathrm{~S}<\mathrm{N}^{*}$ \\
\hline $\begin{array}{l}\text { PICTURE } \\
\text { ARRANGEMENT }\end{array}$ & $7.83 \pm 8.00$ & $6.63 \pm 22.24$ & $9.03 \pm 7.22$ & $16.77 * *$ & $\mathrm{~S}<\mathrm{D}^{*}, \mathrm{~N}^{*}$ \\
\hline $\begin{array}{l}\text { OBJECT. } \\
\text { ASSEMBLY }\end{array}$ & 5.40 & 5.67 & 8.63 & $49.21 * *$ & $\mathrm{~S}<\mathrm{N}^{*}$ \\
\hline
\end{tabular}

**Significance $(\mathrm{p}<.001)$

Table-3 shows the comparison of Depression, schizophrenia and normal groups on WAPIS performance. The groups differed significantly $(\mathrm{p}<.001)$ on all the variables i.e. picture completion, digit symbol, block design, picture arrangement and object assembly. Post Hoc analyses reveals that on all the subtests schizophrenia patients performed poorly than normal and poor than depressed patients on picture completion and picture arrangement.

\begin{tabular}{|c|c|c|c|c|c|}
\hline \multirow[t]{2}{*}{ Variables } & \multicolumn{3}{|l|}{ Groups } & \multirow[t]{2}{*}{$\mathrm{F}$} & \multirow[t]{2}{*}{ Post Hoc } \\
\hline & $\begin{array}{l}\text { Depression } \\
\text { Mean } \pm S D\end{array}$ & $\begin{array}{l}\text { Schizophrenia } \\
\text { Mean } \pm \text { SD }\end{array}$ & $\begin{array}{l}\text { Normal } \\
\text { Mean } \pm \text { SD }\end{array}$ & & \\
\hline Information & $79.83 \pm 9.23$ & $95.43 \pm 20.85$ & $97.20 \pm 7.26$ & 14.36 & $\mathrm{~S}>\mathrm{D}^{*}$ \\
\hline Digit span & $87.20 \pm 8.03$ & $88.30 \pm 7.46$ & $100.93 \pm 4.02$ & 38.47 & $\mathrm{~S}<\mathrm{N}^{*}$ \\
\hline Arithmetic & $82.73 \pm 8.20$ & $86.07 \pm 14.31$ & $97.17 \pm 19.02$ & 8.11 & $\mathrm{~S}<\mathrm{N}^{*}$ \\
\hline Comprehension & $88.93 \pm 22.24$ & $68.97 \pm 8.00$ & $97.57 \pm 7.22$ & 31.71 & $\mathrm{~N}>\mathrm{S}, \mathrm{D}^{*}$ \\
\hline
\end{tabular}


Table-4 showing comparison of Depression, schizophrenia and normal groups on VAIS.

Table-4 shows the comparison of Depression, schizophrenia and normal groups on VAIS performance. The groups differed significantly on all the variables i.e. Information, Digit span, Arithmetic and Comprehension. Post Hoc analyses reveals that on Digit span, Comprehension \& Arithmetic variables schizophrenia patients performed poorly than normal and better than depressed on Information. On comprehension both patient groups performed poorly.

\section{DISCUSSION:}

In order to understand the differences in intellectual functioning of patients of schizophrenia depression and normal controls this study was undertaken. Cognitive functions refer to the ability to attend to things in a selective and focused way, to concentrate over a period of time, to learn new information and skills, to plan, to determine strategies for actions and to execute them, to comprehend language and to use verbal skills for communication and self-expression and to retain information and manipulate it to solve complex problems are examples of mental processes. These are integral part of intelligence. Since cognitive deficits are prominent in schizophrenia and depression both, this study aims to understand the similarities and differences in their VAIS and WAPIS profile, which would help in management of these cases.

In this study, the mean age of Schizophrenia, Depression \& Normal groups didn't differ significantly. But post hoc analyses show that the schizophrenia and depressive groups differed significantly as the patients of depression had higher age mean than that of schizophrenia. This is consistent with the fact that mood disorders start later in age as compared to schizophrenia. On the other socio-demographic variables of all the groups were homogeneous. No significant differences were found among the groups regarding sex, education, marital status, occupation etc.

\section{Comparison of Depression, schizophrenia and normal groups on WAPIS}

The groups differed significantly $(\mathrm{p}<.001)$ on all the variables i.e. picture completion, digit symbol, block design, picture arrangement and object assembly. On all the subtests schizophrenia patients performed poorly than normal and poor than depressed patients on picture completion and picture arrangement.

Schizophrenia patients poor performance than normal on the subtests picture completion, digit symbol, block design, picture arrangement and object assembly is consistent with the earlier findings of significant overall cognitive impairment in this group (Goldberg, Gold, et al., 1995; Green 1996; Green et al., 2000).Object Assembly is a test of motor coordination and control, as are Digit Symbol-Coding and Block Design. These measure the ability to differentiate familiar configuration, and its also involves some anticipation and planning, ability to benefit from sensory - motor feedback, anticipation of relationship among parts, visual - motor organization, simultaneous processing, synthesis, putting things together in a familiar configuration and ability to differentiate familiar configurations. Low scores show visual- motor disorganization, 
concreteness and difficulties with visual concept formation. Both Block Design and Object Assembly are sensitive to right, especially right posterior, lesions (Lezak, 1995; Reiten \& Wolson, 1993).

The picture completion subtest is a measure of visual concentration and is a nonverbal test of general information. It involves discovering consistency and inconsistency by paying close attention to the environment and accessing remote memory, visual alertness, visual recognition and identification, awareness of environmental detail; reality contact, perception of the whole in relation to its parts; visual conceptual ability and visual concentration. Low scores indicate poor concentration and inadequate visual organization. Impulsiveness can often produce lowered performance because the examinee may make quick responses without carefully analyzing the whole picture. Schizophrenia patients poor performance than depressed patients on picture completion indicates poor concentration and inadequate visual organization. Deficits in attention and information processing might be "central" to the disorder [Braff . et al., 1993 ] because these can contribute to deficits in working memory and executive function. Attentional deficits have been found to be robustly associated with deficit syndrome and its persistent (primary) symptom [Ross DE, et al 1997]. Patients with schizophrenia who are highly distractible have been found to have higher levels of formal thought disorder [Perry W,et al 1994 ].

The picture Arrangement subtest is primarily a test of the ability to plan, interpret, and accurately anticipate social events in a given cultural context. Weschsler (1958) stated that the test requires an examinee to use general intelligence in nonverbal social situations. In fact, each of the items requires a person to respond to some practical interpersonal interaction, planning ability, anticipation of consequences, temporal sequencing and time concepts, accurately understanding nonverbal interpersonal situations, visual organization and speed of associating and planning information. Low scores on picture arrangement suggest a paucity of ideas, slow processing of information, a poor sense of humour, difficulty in interpersonal relationship and poor rapport.

Picture Arrangement is some what sensitive to the effects of brain damage, especially for those injuries that disrupt nonverbal social skills (Golden, 1979; Lezak, 1995). Schizophrenia patients also show inability to benefit from nonverbal social cues. There is also some evidence that patients with frontal lobe impairment do poorly on picture arrangement because of their tendency to respond impulsively and without considering the entire problem (Walsh, 1994). These findings are in accordance with the current understanding of the psychopathology of schizophrenia.

\section{Comparison of Depression, schizophrenia and normal groups on VAIS}

The groups differed significantly on all the variables i.e. Information, Digit span, Arithmetic and Comprehension. Schizophrenia patients performed poorly than normal subjects on Digit span, Comprehension \& Arithmetic variables and better than depressed on Information \& both groups performed poorly on comprehension than normal subjects. 
The information subtest sample the type of knowledge that average persons with average opportunities should be able to acquire. It includes the range of general factual knowledge, old learning or schooling, Intellectual curiosity or urge to collect knowledge, Alertness to day-today world and Long - term memory. Performance on the information subtest involves remote memory and alertness to the environment. A high score on this subtest suggests that the examinee has good long-term memory, cultural interests. The comparatively better performance on information subtest in schizophrenia patients is in contrast to earlier findings. As some of the impairments of long-term memory are so severe that they have been said to present a differential deficit in schizophrenia, whereas other memory and learning functions have been found to be relatively preserved compared with healthy people. Episodic or declarative memory, the ability to learn and recall information from previously experienced events [Tulving, 1983], has been proposed to present a differential deficit in schizophrenia [Gold, 1992]. A profile analysis [using a comprehensive neuropsychological battery] of medication -free and medication - naïve patients with schizophrenia showed that memory function was more severely impaired than other cognitive abilities, or sensory and motor function [Saykin et al., 1991; Paulsen et al., 1995]. The effects of institutionalization or long-term neuroleptic medication cannot account for memory impairment. Memory problems in schizophrenia are not due to encoding and retrieval deficits. Relatively mild recognition deficits compared with moderate -to-severe recall disruption have been found in schizophrenia patients [Paulsen et al., 1995],suggesting that information storage in schizophrenia is relatively intact. Encoding of the information has been found to be significantly impaired, however [Gold et al., 1992] patients with schizophrenia benefit less from prompted and cued recall than healthy individuals ; this might be due to the failure to use semantic structure embedded in the information to aid the encoding and the retrieval of the information [Mc Kay et al., 1996]. This failure to use semantic features is not due simply to the lack of strategy use. Even when patients are instructed to cluster the information [eg, by categories], they still do not benefit from the semantic structure embedded in the information that they have to learn. This failure to use a clustering of encoding and retrieval problems [Stone et al., 1998] and has been attributed to impaired executive aspects of working memory [Goldman et al 1994]. The findings of present study may be due to the fact that that all patients of schizophrenia were having low scores on BPRS that means low level of psychopathology at the time of testing. Besides, other aspects of learning and memory in schizophrenia are not impaired to the same extent as semantic and episodic memory systems. Deficits in procedural learning, the ability to learn skills and motor acts, are relatively mild in schizophrenia. There has been some uncertainty as to what extent the Impairment in procedural learning is inherent to the disease process and to what extent it is impaired by the treatment.

Low scores may show superficiality of interests, lack of intellectual curiosity, cultural deprivation, or lack of familiarity (Kaplan et al., 1991; Mittenberg et al., 1989; J. Ryan \& Paul, 1999). Depressive patients scoring low on this subtest may be due to their lack of interest and curiosity during these phases. Again subjective complaints of memory loss are often reported by patients with mood disorders and have been confirmed during neuropsychological assessment of 
declarative memory. Impairments have been reported during the depressed, manic, and euthymic phases of illness in tests of verbal memory, such as story recitation [Zubieta et al 2001] and word list recall [Paradiso et al., 1997; Austin et al 1992; McGrath et al., 1997; Gourovitch et al., 1999].

Comprehension has often been considered to reflect the extent to which an examinee adheres to conventional standards, has benefited from past cultural opportunities, and has a well - developed conscience. It demonstrates practical knowledge, social maturity, knowledge of conventional standards of behaviour, ability to evaluate past experience; that is, proper selection, organization, and emphasis of facts and relationships etc. Formal studies have generally not supported a relationship between Comprehension and various measures of social intelligence (Beebe, Pfiffner \& Mc Burnett, 2000). Low scores, especially if they have four or more subscale points below Vocabulary, might have poor judgement, impulsiveness, and hostility against their environment. Mentally disturbed persons often do poorly on Comprehension, which may be the result of disturbed perceptions, idiosyncratic thinking, impulsiveness, or antisocial tendencies. Both the patient groups performed poorly on comprehension test which is consistent with their psychopathology.

From the above discussion it becomes evident that the three groups differed significantly in intellectual functioning. Hence we reject the null hypothesis that their will be no differences between the groups. Schizophrenia patients have problems of attention and concentration and have inadequate visual organization. They also have paucity of ideas, slow processing of information, a poor sense of humour, difficulty in interpersonal relationship and problem in rapport establishment. Depressive patients perform in a way similar to normal except in comprehension subtest which may be the result of disturbed perceptions, they also have memory problems.

\section{CONCLUSION}

Schizophrenia patients suffer from significant overall cognitive impairment; Have low concentration and inadequate visual organization. They also have paucity of ideas, slow processing of information, a poor sense of humour, difficulty in interpersonal relationship and problem in rapport establishment. Depressive patients perform in a way similar to normal except in comprehension subtest which may be the result of disturbed perceptions, they also have memory problems. 


\section{REFERENCES}

1. Allen DN, Goldstein G,Warnick E.A consideration of neuropsychologically normal schizophrenia, J Int Neuropsychol Soc 2003;9:56-63.

2. American psychiatric Association (APA). Diagnostic and statistical manual of mental disorders. $4^{\text {th }}$ edition (DSM-IV). Washington (DC): American Psychiatric Association; 1994.

3. Andreasen N, Black D. introductory textbook of psychiatry. Washington (DC): American Psychiatric Press; 1995.

4. Calev A,korian Y, Shapira B, et al.Verbal and nonverbal recall by depressed and euthymic affective patients.Psychol Med 1986;16(4):789-94.

5. Censits DM,Ragland JD, gur RC, et al. Neuropsychological evidence supporting a neurodevelopmental model of schizophrenia: a longitudinal study. Schizoph Res 1997; 24:289-98.

6. Cirillo MA, Seidman L.J.Verbal declarative memory dysfunction in schizophrenia: from clinical assessment to genetics and brain mechanisms. Neuropsychol Rev 223;13:43-77.

7. Dobbins C, Russel EW. Left temporal lobe brain damage pattern on the Wechsler Adult Intelligence Scale. Journal of clinical psychology 1990;46: 863-68.

8. Frith CD, Leary J, Cahill C, et al. Performance on psychological tests: demographic and clinical correlates of these results. Br J Psychiatry 1991; 159:26-9.

9. Goldberg E, Seidman LJ, Higher cortical function in normals and in Schizophrenia : a selective review 1991; 5: 553-597.

10. Hamiltin M, "Development of a rating scale for primary depressive illness." Br J Soc Clin Psychol 1967; 6: 278-296.

11. Hamilton MA, Rating Scale for Depression.J Neural Neursing Psychuatry 1960; 23: 5662.

12. Heinrichs RW, Zakazanis KK. Neurocognitive deficit in schizophrenia: a quantitative review of the evidence. Neuropsychology 1998; 12:426-45.

13. Hove AL, Riordan H, O'Donnell DW, et al. Neuropsychological functioning of first episode schizophrenia patients. Am J psychiatry 1992; 149:898-903.

14. Kaplan E, Fein D, Morris R, Delis D.WAIS-R as a Neuropsychological instrument. San Antonio, TX: Psychological Corporation 1991.

15. Kaufman AS, Lictenberger EO. Essentials of WAIS-III assessment. New York: Wiley 1999.

16. La Rue A, Jaravik L R. Cognitive function and prediction of dementia in old age. International Journal of Aging and Human Development 1987; 25:78-89.

17. Lezak MD. Neuropsychological assessment ( $3^{\text {rd }}$ ed.). New York: Oxford University press 1995. 2001; 48:45-55.

18. WAIS-III in a sample of persons with substance abuse disorders. International journal of Neurosciences 1999; 98:131-40. 
19. Verma S K, Wig N N, Pershad D “ P .G .I. Health Questionnaire N-1.”National Psychological Corporation Agra1985.

20. Waldo et al, 1994 In Cognitive Dysfunction in first episode Schizophrenia. Industrial Psychiatry Journal 2003;12: 15-22.

21. Walsh k.Neuropsychological assessment of patients with memory disorders. In Toyz S, Byrne D, Gilandas A, (Eds.), Neuropsychology, in clinical practice (pp.107-27). New York: Academic Press 1994.

22. Wechsler D. Measurement and Appraisal of Adult Intelligence. Malhotra,Newyork: William \& William 1958.

23. Wechsler D. The measurement and appraisal of adult intelligence $\left(4^{\text {th }}\right.$ ed) Baltimore : Williams \& Wilkins 1958.

24. Wechsler D. Wechsler Adult Intelligence scale-revised (WAIS-R) manual. New York: The Psychological Corporation; 1981.

25. Weschler D.Manual for the Wechsler Adult Intelligence,Scale. New York: Psychological Corporation.

26. Zubieta JK, Huguelet P, O’Neil RL, et al. Cognitive function in euthymic bipolar I disorder. Psychiatry Res 2001; 102 (1):9-20. 